\title{
On a Coefficient Conjecture of Brannan
}

\author{
Roger W. Barnard, Kent Pearce, and William Wheeler
}

\begin{abstract}
In 1972, D.A. Brannan conjectured that all of the odd coefficients, $a_{2 n+1}$, of the power series $(1+x z)^{\alpha} /(1-z)$ were dominated by those of the series $(1+z)^{\alpha} /(1-z)$ for the parameter range $0<\alpha<1$, after having shown that this was not true for the even coefficients. He verified the case when $2 n+1=3$. The case when $2 n+1=5$ was verified in the mid-eighties by J.G. Milcetich. In this paper, we verify the case when $2 n+1=7$ using classical Sturm sequence arguments and some computer algebra.
\end{abstract}

1980 AMS Subject Classification: 30C50

Keywords: coefficient conjecture, computer algebra 


\section{Introduction.}

For $k \geq 2$ let $V_{k}$ denote the class of locally univalent analytic functions

$$
f(z)=z+a_{2} z^{2}+a_{3} z^{3}+\cdots
$$

which map $|z|<1$ conformally onto a domain whose boundary rotation is at most $k \pi$. (See $[\mathrm{Pa}]$ for the definition and basic properties of the class $V_{k}$.)

The function

$$
f_{k}(z)=\frac{1}{k}\left[\left(\frac{1+z}{1-z}\right)^{\frac{k}{2}}-1\right]=\sum_{n=1}^{\infty} A_{n} z^{n}
$$

belongs to $V_{k}$. The coefficient conjecture for the class $V_{k}$ was that for a function (1) in $V_{k}$ that

$$
\left|a_{n}\right| \leq A_{n}, \quad(n \geq 1) .
$$

This conjecture was verified for $n=2$ by Pick (see [Le]), for $n=3$ by Lehto [Le] in 1952 and for $n=4$ by Schiffer and Tammi [ScTa] in 1967, Lonka and Tammi [LoTa] in 1968 and Brannan [Br1] in 1969.

Using extreme point theory arguments, Brannan, Clunie and Kirwan [BrClKi] showed in 1973 that (2) can be reduced to showing that for

$$
\Phi(\alpha, x ; z)=\left(\frac{1+x z}{1-z}\right)^{\alpha}=\sum_{n=1}^{\infty} B_{n}(\alpha, x) z^{n}
$$

that

$$
\left|B_{n}(\alpha, x)\right| \leq B_{n}(\alpha, 1), \quad(n \geq 1)
$$

for $\alpha \geq 1,|x|=1$. Brannan, Clunie and Kirwan showed that (3) holds for $1 \leq n \leq 13$, which implies (2) for $2 \leq n \leq 14$.

In 1972 Aharonov and Friedland [AhFr] considered a related coefficient inequality. Let

$$
\Psi(\alpha, x ; z)=\frac{(1+x z)^{\alpha}}{1-z}=\sum_{n=1}^{\infty} A_{n}(\alpha, x) z^{n}
$$


In $[\mathrm{AhFr}]$ it was shown, by a long technical argument, that

$$
\left|A_{n}(\alpha, x)\right| \leq A_{n}(\alpha, 1), \quad(n \geq 1)
$$

for $\alpha \geq 1,|x|=1$, which implies (3) and, hence, by the work in [BrClKi], also implies (2). Later, in 1973 Brannan [Br2] gave a short, elegant proof that (4) holds for $\alpha \geq 1,|x|=1$.

In [Br2] Brannan also considered the question about whether (4) holds for $0<\alpha<1,|x|=1$. He showed there the unexpected result that for each $\alpha, 0<\alpha<1$, there exists an $n_{\alpha}$ such that

$$
\max _{|x|=1} \operatorname{Re} A_{2 n}(\alpha, x)>A_{2 n}(\alpha, 1)
$$

for $n>n_{\alpha}$, that is, that (4) fails for even coefficients when $0<\alpha<1$.

Brannan showed, using an inequality for quadratic trigonometric polynomials, that

$$
\left|A_{3}(\alpha, x)\right| \leq A_{3}(\alpha, 1)
$$

for $0<\alpha<1$ and he conjectured, based on numerical data, that

\section{Brannan's Conjecture}

$$
\left|A_{2 n+1}(\alpha, x)\right| \leq A_{2 n+1}(\alpha, 1), \quad(n \geq 1)
$$

for $0<\alpha<1,|x|=1$.

Brannan's conjecture has been verified for $n=2$, that is, for $2 n+1=5$, by Milcetich [Mi], who employed a lengthy argument based on a result of Brown and Hewitt [BrHe] for positive trigonometric sums.

In this paper, we will establish Brannan's conjecture for $n=3$, that is, for $2 n+1=7$. The method we will employ is based largely on (i) a judicious rearrangement of the coefficients $A_{n}(\alpha, x)$ over carefully chosen subintervals of $(0,1)$, the domain of $\alpha$, (ii) an application of Sturm sequences to verify the nonnegativity of those rearrangements and (iii) using a computer algebra 
program (in this case Maple) to generate the coefficients $A_{n}(\alpha, x)$ and the Sturm sequences.

\section{Section 1.}

Brannan's coefficient inequality (6) is equivalent to

$$
A_{2 n+1}^{2}(\alpha, 1)-\left|A_{2 n+1}(\alpha, x)\right|^{2} \geq 0
$$

for $0<\alpha<1,|x|=1$. We will let $F_{2 n+1}(\alpha, x)$ denote the left-hand side of (7) and we will show for $2 n+1=7$ that $F_{2 n+1}(\alpha, x) \geq 0$.

We note that

$$
\begin{aligned}
\frac{(1+x z)^{\alpha}}{(1-z)} & =\sum_{n=0}^{\infty} \frac{(-\alpha)_{n}(-1)^{n} x^{n}}{n !} z^{n} \sum_{n=0}^{\infty} z^{n} \\
& =\sum_{n=0}^{\infty} \sum_{k=0}^{n} \frac{(-\alpha)_{k}(-1)^{k} x^{k}}{k !} z^{n} \\
& =\sum_{n=0}^{\infty} A_{n}(\alpha, x) z^{n},
\end{aligned}
$$

where $(a)_{k}$ denotes the Pockhammer symbol, which is defined as

$$
(a)_{k}=\left\{\begin{array}{cc}
1 & k=0 \\
a(a+1) \cdots(a+k-1) & k>0
\end{array} .\right.
$$

Hence, we can write $F_{N}(\alpha, x)$ as

$$
\begin{aligned}
F_{N}(\alpha, x)= & \sum_{k=0}^{N} \frac{(-\alpha)_{k}(-1)^{k}}{k !} \sum_{k=0}^{N} \frac{(-\alpha)_{k}(-1)^{k}}{k !}- \\
& \sum_{k=0}^{N} \frac{(-\alpha)_{k}(-1)^{k} x^{k}}{k !} \sum_{k=0}^{N} \frac{(-\alpha)_{k}(-1)^{k} \bar{x}^{k}}{k !}
\end{aligned}
$$




$$
\begin{aligned}
= & \sum_{k=0}^{2 * N} \sum_{j=0}^{k} \frac{(-\alpha)_{j}(-\alpha)_{k-j}(-1)^{k} \delta_{j} \delta_{k-j}}{k !(k-j) !}- \\
& \sum_{k=0}^{2 * N} \sum_{j=0}^{k} \frac{(-\alpha)_{j}(-\alpha)_{k-j}(-1)^{k} \delta_{j} \delta_{k-j} x^{2 * j-k}}{k !(k-j) !}
\end{aligned}
$$

where

$$
\delta_{j}=\left\{\begin{array}{cc}
1 & 0 \leq j \leq N \\
0 & N+1 \leq j \leq 2 * N
\end{array}\right.
$$

Since $F_{N}(\alpha, x)$ is real, we can write, setting $x=e^{i \theta}$,

$$
\begin{aligned}
F_{N}(\alpha, x)= & \sum_{k=0}^{2 * N} \sum_{j=0}^{k} \frac{(-\alpha)_{j}(-\alpha)_{k-j}(-1)^{k} \delta_{j} \delta_{k-j}}{k !(k-j) !}- \\
& \sum_{k=0}^{2 * N} \sum_{j=0}^{k} \frac{(-\alpha)_{j}(-\alpha)_{k-j}(-1)^{k} \delta_{j} \delta_{k-j} \cos ((2 * j-k) \theta)}{k !(k-j) !}
\end{aligned}
$$

The following Maple Procedure can be used to generate the coefficients of $F_{N}(\alpha, x)$, where $x=e^{i \theta}$,

\section{Procedure 1}

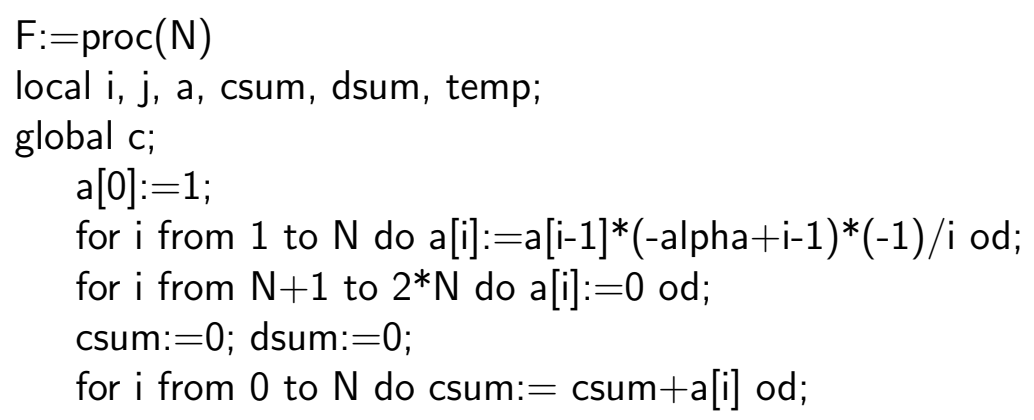




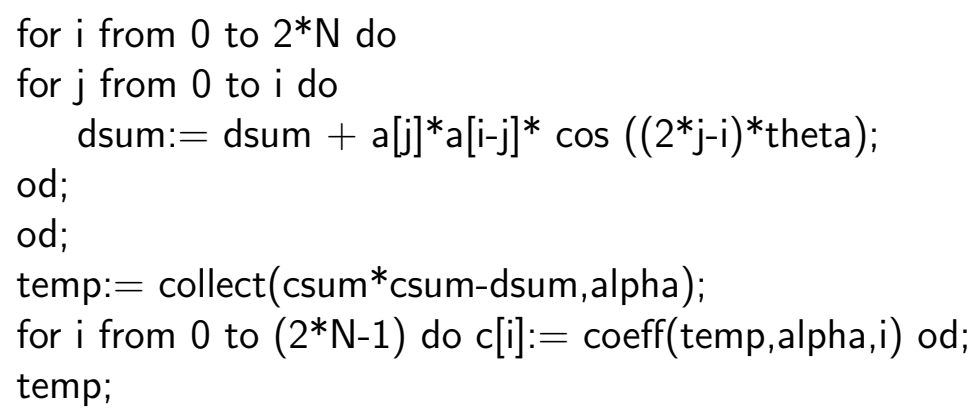

Using Procedure 1, we obtain for $N=7$ that $F_{7}(\alpha, x)=\sum_{k=1}^{13} c_{k}(\theta) \alpha^{k}$ where each $c_{k}(\theta)$ is a trigonometric polynomial of the form $c_{k}(\theta)=\sum_{j=0}^{7} a_{k j} \cos (j \theta)$ with rational coefficients $a_{k j}$. We will show that $F_{7}(\alpha, x) \geq 0$ for $0<\alpha<1$ by subdividing the domain of $\alpha$ into subintervals $0<\alpha \leq t_{0}$ and $t_{0}<\alpha<1$, where $t_{0}=2 / 5$. We will show that $F_{7}(\alpha, x) \geq 0$ on each subinterval.

First for the case $0<\alpha \leq t_{0}$ we will show the following:

$$
\begin{aligned}
& c_{1}(\theta) \geq 0, \quad \frac{7}{10} c_{1}(\theta)+c_{2}(\theta) t_{0} \geq 0 \\
& \frac{1}{10} c_{1}(\theta)+c_{3}(\theta) t_{0}^{2} \geq 0, \quad \frac{1}{10} c_{1}(\theta)+c_{3}(\theta) t_{0}^{2}+c_{4}(\theta) t_{0}^{3} \geq 0, \\
& \frac{1}{5} c_{1}(\theta)+c_{5}(\theta) t_{0}^{4} \geq 0, \quad \frac{1}{5} c_{1}(\theta)+c_{5}(\theta) t_{0}^{4}+c_{6}(\theta) t_{0}^{5} \geq 0, \\
& c_{7}(\theta) \geq 0, \quad c_{7}(\theta)+c_{8}(\theta) t_{0} \geq 0 \\
& c_{9}(\theta) \geq 0, \quad c_{9}(\theta)+c_{10}(\theta) t_{0} \geq 0 \\
& c_{11}(\theta) \geq 0, \quad c_{11}(\theta)+c_{12}(\theta) t_{0} \geq 0 \\
& c_{13}(\theta) \geq 0 .
\end{aligned}
$$

It will follow then that for $0<\alpha \leq t_{0}$ we have 


$$
\begin{aligned}
F_{7}(\alpha, x) & =\left[\frac{7}{10} c_{1}(\theta)+c_{2}(\theta) \alpha\right] \alpha+\left[\frac{1}{10} c_{1}(\theta)+c_{3}(\theta) \alpha^{2}+c_{4}(\theta) \alpha^{3}\right] \alpha \\
& +\left[\frac{1}{5} c_{1}(\theta)+c_{5}(\theta) \alpha^{4}+c_{6}(\theta) \alpha^{5}\right] \alpha+\left[c_{7}(\theta)+c_{8}(\theta) \alpha\right] \alpha^{7} \\
& +\left[c_{9}(\theta)+c_{10}(\theta) \alpha\right] \alpha^{9}+\left[c_{11}(\theta)+c_{12}(\theta) \alpha\right] \alpha^{11} \\
& +c_{13}(\theta) \alpha^{13} \geq 0
\end{aligned}
$$

The inequalities (9) imply (10) because they imply that each of the terms in brackets in (10) are non-negative. The non-negativity of the bracketed terms of the form $\left[c_{i}(\theta)+c_{i+1}(\theta) \alpha\right]$ follows from (9.1), (9.4), (9.5) and (9.6) because the terms are linear in $\alpha$ and, hence they take their minimum at either $\alpha=0$ or else at $\alpha=t_{0}$.

Since $\left[c_{3}(\theta)+c_{4}(\theta) \alpha\right]$ is linear in $\alpha$, it takes its minimum at either $\alpha=0$ or else at $\alpha=t_{0}$. Thus, we have

$$
\frac{1}{10} c_{1}(\theta)+\left(c_{3}(\theta)+c_{4}(\theta) \alpha\right) \alpha^{2} \geq \frac{1}{10} c_{1}(\theta)+\min _{0<s \leq t_{0}}\left\{c_{3}(\theta)+c_{4}(\theta) s\right\} \alpha^{2} .
$$

The right-hand side of (11) is linear in $\alpha^{2}$, and hence takes its minimum at either $\alpha=0$ or else at $\alpha=t_{0}$. Therefore, the right-hand side of (11) is non-negative by (9.2).

Similarly, since $\left[c_{5}(\theta)+c_{6}(\theta) \alpha\right]$ is linear in $\alpha$, it takes its minimum at either $\alpha=0$ or else at $\alpha=t_{0}$. Thus, we have

$$
\frac{1}{5} c_{1}(\theta)+\left(c_{5}(\theta)+c_{6}(\theta) \alpha\right) \alpha^{4} \geq \frac{1}{5} c_{1}(\theta)+\min _{0<s \leq t_{0}}\left\{c_{5}(\theta)+c_{6}(\theta) s\right\} \alpha^{4} .
$$

The right-hand side of (12) is linear in $\alpha^{4}$, and hence takes its minimum at either $\alpha=0$ or else at $\alpha=t_{0}$. Therefore, the right-hand side of (12) is non-negative by (9.3).

Thus, to complete the case $0<\alpha \leq t_{0}$ we will need to establish (9). We will transform each of the trigonometric coefficients $c_{i}(\theta)$, which are polynomials in $\cos (n \theta)$, to polynomials in $\cos \theta$ and then by a change of variable to polynomials $e_{i}(x),-1 \leq x \leq 1$. To verify the non-negativity of the linear combinations of trigonometric coefficients $c_{i}(\theta)$ specified in (9), we will 
establish the non-negativity of the same linear combinations of polynomials $e_{i}(x)$.

The following two Maple procedures can be used to: (i) transform the trigonometric coefficients $c_{i}(\theta)$ to the polynomials $e_{i}(x)$; and (ii) compute the number of roots of a polynomial $\mathrm{p}$ on the interval $(-1,1]$ via a Sturm sequence argument.

\section{Procedure 2}

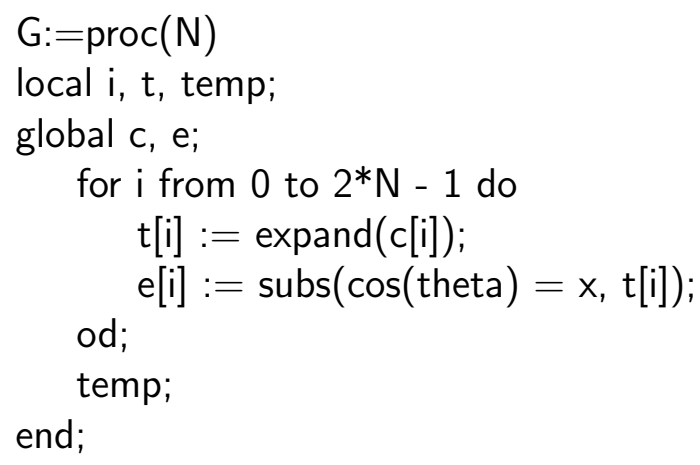

Here $\mathbf{N}$ is chosen the same as in Procedure 1.

\section{Procedure 3}

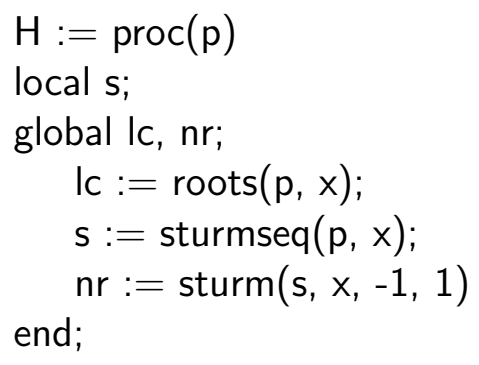

The library call readlib(sturm) must be loaded prior to applying the procedure. 
If the polynomial $e(x)$, created from linear combinations of the $e_{i}(x)$ after applying Procedure 2, is assigned to the variable $\mathrm{p}$, then Procedure 3 will compute both the number of roots of $e(x)$ on the interval $(-1,1]$ and the location of the rational roots of $e(x)$. We will see that the conclusion of this application of Procedure 3 is that the polynomial $e(x)$ is non-negative on $[-1,1]$ with $e(x)=0$ only for $x=1$. This check can be confirmed for each of the polynomials $e(x)$ which arise as linear combinations of the polynomials $e_{i}(x)$, where the linear combinations are specified as in (9), and, thus, complete the case $0<\alpha \leq t_{0}$.

To illustrate the utility of using computer algebra software to establish the inequalities in (9) we will explicitly demonstrate the process for the inequality (9.1). Applying Procedure 1 to compute the trigonometric coefficients $c_{i}(\theta)$ of $F_{7}(\alpha, x)$, we obtain

$$
\begin{aligned}
c_{1}(\theta) & =-\frac{2}{5} \cos (5 \theta)+\cos (2 \theta)-\frac{2}{3} \cos (3 \theta)+\frac{1}{3} \cos (6 \theta) \\
& +\frac{1}{2} \cos (4 \theta)-2 \cos (\theta)-\frac{2}{7} \cos (7 \theta)+\frac{319}{210}
\end{aligned}
$$

From Procedure 2 we obtain

$$
\begin{aligned}
e(x) & =e_{1}(x)=\frac{128}{5} x^{5}-\frac{32}{3} x^{3}+4 x^{2}+\frac{24}{35} \\
& +\frac{32}{3} x^{6}-12 x^{4}-\frac{128}{7} x^{7} .
\end{aligned}
$$

Then, Procedure 3 yields

$$
\begin{aligned}
& >\mathrm{H}(\mathrm{p}) ; \\
& >\text { lc }
\end{aligned}
$$

$$
[[1,1]]
$$

The value returned by the procedure call $H(p)$ is the number of roots of $p$ on $(-1,1]$ and the value returned by $\mathrm{lc}$ is the interval location of the rational roots of $\mathrm{p}$. 
For the second half of (9.1), we have

$$
\begin{aligned}
\frac{7}{10} c_{1}(\theta)+c_{2}(\theta) t_{0} & =\frac{128}{525} \cos (5 \theta)-\frac{37}{210} \cos (2 \theta)+\frac{451}{1575} \cos (3 \theta) \\
& -\frac{292}{1575} \cos (60)-\frac{197}{700} \cos (4 \theta)-\frac{5}{7} \cos (\theta)+\frac{2}{25} \cos (7 \theta)+\frac{523}{700}
\end{aligned}
$$

From Procedure 2 we obtain

$$
\begin{aligned}
e(x)=\frac{7}{10} e_{1}(x)+e_{2}(x) t_{0} & =-\frac{2656}{525} x^{5}+\frac{236}{315} x^{3}-\frac{151}{105} x^{2} \\
& +\frac{1303}{1575}-\frac{9344}{1575} x^{6}+\frac{698}{105} x^{4}+\frac{128}{25} x^{7}-\frac{32}{35} x
\end{aligned}
$$

Then, Procedure 3 yields

$$
\begin{aligned}
& >\mathrm{H}(\mathrm{p}) ; \\
& >\mathrm{lc} \\
&
\end{aligned} \quad[[1,1]]
$$

We have that each linear combination $e(x)$ has only one root on $(-1,1]$ and that root is at $x=1$. Since we can explicitly observe that each $e(0)>0$, we can conclude that each $e(x)$ is non-negative on $[-1,1]$. Therefore, we have that (9.1) holds.

For the case $t_{0}<\alpha<1$ we make the substitution $\alpha=\beta+t_{0}$. Then, we have $F_{7}(\alpha, x)=G_{7}(\beta, x)=\sum_{k=0}^{13} d_{k}(\theta) \beta^{k}$ where each $d_{k}(\theta)$ is a trigonometric polynomial of the form $d_{k}(\theta)=\sum_{j=0}^{7} b_{k j} \cos (j \theta)$ and $0<\beta<t_{1}=3 / 5$. It will suffice to show for this case that

$$
\begin{aligned}
d_{0}(\theta) & \geq 0, \quad d_{0}(\theta)+d_{2}(\theta) t_{1}^{2} \geq 0 \\
d_{1}(\theta) & \geq 0, \quad \frac{2}{3} d_{1}(\theta)+d_{5}(\theta) t_{1}^{4} \geq 0 \\
\frac{1}{3} d_{1}(\theta)+d_{6}(\theta) t_{1}^{5} & \geq 0, \quad \frac{1}{3} d_{1}(\theta)+d_{6}(\theta) t_{1}^{5}+d_{8}(\theta) t_{1}^{7} \geq 0
\end{aligned}
$$




$$
\begin{gathered}
d_{3}(\theta) \geq 0, d_{3}(\theta)+d_{4}(\theta) t_{1} \geq 0 \\
d_{7}(\theta) \geq 0 \\
d_{9}(\theta) \geq 0, \quad d_{9}(\theta)+d_{10}(\theta) t_{1} \geq 0, \\
d_{11}(\theta) \geq 0, \quad d_{11}(\theta)+d_{12}(\theta) t_{1} \geq 0 \\
d_{13}(\theta) \geq 0 .
\end{gathered}
$$

For then, it will follow that for $0<\beta<t_{1}$ we have

$$
\begin{aligned}
G_{7}(\beta, x) & =\left[d_{0}(\theta)+d_{2}(\theta) \beta^{2}\right]+\left[\frac{2}{3} d_{1}(\theta)+d_{5}(\theta) \beta^{4}\right] \beta \\
& +\left[\frac{1}{3} d_{1}(\theta)+d_{6}(\theta) \beta^{5}+d_{8}(\theta) \beta^{7}\right] \beta+\left[d_{3}(t)+d_{4}(\theta) \beta\right] \beta^{3} \\
& +d_{7}(\theta) \beta^{7}+\left[d_{9}(\theta)+d_{10}(\theta) \beta\right] \beta^{9}+\left[d_{11}(\theta)+d_{12}(\theta) \beta\right] \beta^{11} \\
& +d_{13}(\theta) \beta^{13} \geq 0
\end{aligned}
$$

The inequalities (13) imply (14) because they imply that each of the bracketed terms in (14) are non-negative for $0<\beta<t_{1}$. Procedure 2 can be adapted (by changing the global variable $\mathrm{c}$ to $\mathrm{d}$ ) so that it can be applied to each of the trigonometric coefficients $d_{i}(\theta)$ to generate new polynomials $e_{i}(x)$. Then, Procedure 3 can be applied to each of the (transformed) linear combinations specified in (13) to verify (14) and thus, complete the case $0<\beta<t_{1}$.

\section{Remarks.}

1. We have verified the above constructions alternately using Mathematica for the computer algebra component of the construction.

2. The above process can be applied to $F_{3}(\alpha, x)$ and $F_{5}(\alpha, x)$ to give relatively straight-forward proofs of two cases of Brannan's conjecture (6), specifically, the cases $2 n+1=3$ and $2 n+1=5$. In the latter case, the proof subdivides the interval $0<\alpha<1$ into two cases $0<\alpha \leq 2 / 5$ and $2 / 5<\alpha<1$. The argument here is substantially simpler than Milcetich's proof. 
3. This technique for verifying Brannan's conjecture (6) for the case $2 n+1=7$ can be applied to an alternate, but closely related coefficient inequality. If in the series representation for $F_{N}(\alpha, x)$ in (8) the summation is extended to infinity, that is, if we write

$$
\begin{aligned}
F_{N}(\alpha, x)= & \sum_{k=0}^{\infty} \sum_{j=0}^{k} \frac{(-\alpha)_{j}(-\alpha)_{k-j}(-1)^{k} \delta_{j} \delta_{k-j}}{k !(k-j) !}- \\
& \sum_{k=0}^{\infty} \sum_{j=0}^{k} \frac{(-\alpha)_{j}(-\alpha)_{k-j}(-1)^{k} \delta_{j} \delta_{k-j} \cos ((2 * j-k) \theta)}{k !(k-j) !}
\end{aligned}
$$

and where again

$$
\delta_{j}=\left\{\begin{array}{ll}
1 & 0 \leq j \leq N \\
0 & N+1 \leq j
\end{array},\right.
$$

then one can define the partial sums

$$
\begin{aligned}
F_{N}^{m}(\alpha, x)= & \sum_{k=0}^{m} \sum_{j=0}^{k} \frac{(-\alpha)_{j}(-\alpha)_{k-j}(-1)^{k} \delta_{j} \delta_{k-j}}{k !(k-j) !}- \\
& \sum_{k=0}^{m} \sum_{j=0}^{k} \frac{(-\alpha)_{j}(-\alpha)_{k-j}(-1)^{k} \delta_{j} \delta_{k-j} \cos ((2 * j-k) \theta)}{k !(k-j) !}
\end{aligned}
$$

Wheeler $[\mathrm{Wh}]$ considered the partial sums $F_{N}^{m}(\alpha, x)$. He showed that these partial sums have many properties which are analogous to the coefficient sums $F_{N}(\alpha, x)$. Specifically, he showed there that for each $\alpha, 0<\alpha<1$, there exists an $m_{\alpha}$ such that

$$
\max _{|x|=1} F_{N}^{2 * m}(\alpha, x)<0
$$

for $m>m_{\alpha}$. Furthermore, he devised the computer algebra technique described above, and applied it to show that for $m=1,3,5$ and 7 ,

$$
F_{N}^{m}(\alpha, x) \geq 0
$$

for $0<\alpha<1$. 


\section{References}

[AhFr] D. Aharonov and S. Friedland, On an inequality connected with the coefficient conjecture for functions of bounded boundary rotation, Ann. Acad. Sci. Fenn. Ser. A 1 Math. 542 (1972), 14 pp.

[Br1] D.A. Brannan, On functions of bounded boundary rotation II, Bull. London Math Soc. 1 (1969), 321-322.

[Br2] D.A. Brannan, On coefficient problems for certain power series, Symposium on Complex Analysis, Canterbury, (1973) eds. J.G. Clunie \& W.K. Hayman. London Math. Soc. Lecture Note Series 12 (1974), 1727.

[BrClKi] D.A. Brannan, J.G. Clunie and W.E. Kirwan, On the coefficient problem for functions of bounded rotation, Ann. Acad. Sci. Fenn. Ser. A 1 Math. 543 (1973), 18 pp.

[BrHe] G. Brown and E. Hewitt, A class of positive trigonometric sums, Math. Ann. 268 (1984), 91-122.

[Le] O. Lehto, On the distortion of conformal mappings with bounded boundary rotation, Ann. Acad. Sci. Fenn. Ser. A 1 Math. Phys. 124 (1952), 14 pp.

[LoTa] H. Lonka and H. Tammi, On the use of step-functions in extremal problems of the class with bounded boundary rotation, Ann. Acad. Sci. Fenn. Ser. A 1418 (1968), 18 pp.

[Mi] J.G. Milcetich, On a coefficient conjecture of Brannan, J. Math. Anal. Appl. 139 (1989), 515-522. 
[Pa] V. Paatero, Über die konforme Abbildung von Gebieten deren Ränder von beschränkter Drehung sind, Ann. Acad. Sci. Fenn. Ser. A 33 (1931), 77 pp.

[ScTa] M. Schiffer and O. Tammi, On the fourth coefficient of univalent functions with bounded boundary rotation, Ann. Acad. Sci. Fenn. Ser. A 396 (1967), $26 \mathrm{pp}$.

[Wh] W. Wheeler, Properties of partial sums of certain special functions in complex variables, Thesis, Texas Tech University, 1995.

Department of Mathematics, Texas Tech University, Lubbock, TX 79409

E-mail: barnard@math.ttu.edu, pearce@math.ttu.edu, bwheeler@math.ttu.edu. 